\title{
EXISTENCE OF NON-TRIVIAL DEFORMATIONS OF INSEPARABLE ALGEBRAIC EXTENSION FIELDS II*
}

\author{
Dedicated to Professor K. Noshiro on his 60 th birthday \\ HIROSHI KIMURA
}

Let $K$ be an extension of a field $k$, and $p$ denotes the characteristic. It was proved by $\mathbf{M}$. Gerstenhaber ([1]) that if $K$ is separable over $k$, then it is rigid and it was conjectured in [1] that, if $K$ is not separable over $k$, then it is not rigid. We studied in [4] the above conjecture in certain special case. In this note we shall extend the results of [4] to inseparable algebraic extension fields.

1. Preliminaries. Let $K$ be an extension fields of a field $k$ of characteristic $p$, and $V$ be the underlying vector space over $k$. Let $R$ and $S$ denote the power series ring $k[[t]]$ over $k$ in one variable $t$ and its quotient field $k((t))$ and $V_{s}$ be $V \otimes_{k} S$.

Let a bilinear mapping $f_{t}: V_{s} \times V_{s} \longrightarrow V_{s}$ expressible in the form

$$
f_{t}(a, b)=a b+t F_{1}(a, b)+t^{2} F_{2}(a, b)+\cdots,
$$

where $F_{i}$ is a bilinear mapping defined over $k$, be a one-parameter family of deformations of $K$ considered as a commutative $k$-algebra.

Following [1], we say that $f_{t}$ is trivial if there is a non-sigular linear mapping $\Phi_{t}$ of $V_{s}$ onto itself of the form

$$
\Phi_{t}(a)=a+t \varphi_{1}(a)+t^{2} \varphi_{2}(a)+\cdots,
$$

where $\varphi_{i}$ is a linear mapping defined over $k$, such that $f_{t}(a, b)=\Phi_{t}^{-1}\left(\Phi_{t} a \cdot \Phi_{t} b\right)$. $K$ is rigid if and only if there is no non-trivial one-parameter family of deformations of $K$.

From now on, throughout this note, we assume $p \neq 0$.

Received April 21, 1967.

* This work was supported by The Sakkokai Foundation. 
It is known ([1]) that, for any derivation $\varphi$ of $K$, there exists a one-parameter family $f_{t}$ of deformations of $K$ such that

$$
f_{t}(a, b)=a b+t F_{1}(a, b)+t^{2} F_{2}(a, b)+\cdots,
$$

where $F_{1}=S q_{p} \varphi=\frac{1}{p} \delta \varphi^{p}=\sum_{i=1}^{p-1} \frac{1}{p}{ }_{p} C_{i} \varphi^{p-i} \cup \varphi^{i} \quad(\delta$ denotes the coboundary operator and $U$ denotes the cup product).

2. In this section we shall prove the following lemma and its corollary.

Lemma 1. Let $R$ be the polynomial ring $k[y]$ and $T$ the non-commutative polynomial ring $R\left[x_{1}, \cdots, x_{s}\right]$. Let $x_{r}^{\prime}$ be the mapping of the set of positive integers into $T$ satisfying the following conditions;

1) $x_{r}^{\prime}(1)=x_{r}$

2) $x_{1}^{\prime}(n)=n x_{1} y^{n-1}$.

3) $x_{r}^{\prime}(n)=x_{r} y^{n-1}+x_{r}^{\prime}(n-1) y+\sum_{i=1}^{r-1} x_{i} x_{r-i}^{\prime}(n-1)$, for $r \geqq 2$.

Then, for $r \geqq 2$,

$$
x_{r}^{\prime}(n)=n x_{r} y^{n-1}+\sum_{n} C_{\Sigma i_{j}} x_{r_{1}}^{i_{1}} \cdots x_{r_{n}^{h}}^{i_{n}} y^{n-\Sigma i_{j}},
$$

where the sum is taken over all sets $\left\{r_{1}, \cdots, r_{h} ; i_{1}, \cdots, i_{h}\right\}$ such that $\sum_{j=1}^{h} r_{j} i_{k}=r$, $2 \leqq \sum_{j=1}^{h} i_{j} \leqq n$ and $1 \leqq r_{j}<r$.

Proof. We shall prove this by induction on $r$ and $n$.

1) The case $r=2$. If $n=2$, then the lemma is trivial. If $n>2$, then

$$
\begin{aligned}
x_{2}^{\prime}(n) & =x_{2} y^{n-1}+x_{2}^{\prime}(n-1) y+x_{1} x_{1}^{\prime}(n-1) \\
& =x_{2} y^{n-1}+\left\{(n-1) x_{2} y^{n-2}+{ }_{n-1} C_{2} x_{1}^{2} y^{n-3}\right\} y \\
& +(n-1) x_{1}^{2} y^{n-2} \\
& =n x_{2} y^{n-2}+{ }_{n} C_{2} x_{1}^{2} y^{n-2}
\end{aligned}
$$

2) The case $r>2$.

$$
x_{r}^{\prime}(2)=2 x_{r} y+\sum_{i=1}^{r-1} x_{i} x_{r-i} .
$$

We assume $n>2$. Then 


$$
\begin{aligned}
& \sum_{\substack{\sum r_{r_{i j}=r} \\
2 \leqq \Sigma i_{j} \leqq n-1}}{ }_{n-1} C_{\Sigma i}, x_{r_{1}}^{i_{1}} \cdots x_{r_{n}^{n}}^{i_{n}} y^{n-\Sigma i}, \\
& 1 \leqq r_{j}<r \\
& =\sum_{i=1}^{r-1} x_{r-i}\left\{_{n-1} C_{2} x_{i} y^{n-2}\right. \\
& \left.+\sum_{\substack{\sum r_{j_{i} i_{j}=i}=i \\
2 \leqq \sum i_{j} \leqq n-2 \\
1 \leqq r_{j}<i}}{ }_{n-1} C_{\Sigma i_{j}+1} x_{r_{1}}^{i_{1}} \cdots x_{r_{n}^{n}}^{i_{n}} y^{r^{n}-\Sigma i_{j}-1}\right\},
\end{aligned}
$$

and

$$
\begin{aligned}
& \sum_{i=1}^{r-1} x_{r-i} x_{i}^{\prime}(n-1)
\end{aligned}
$$

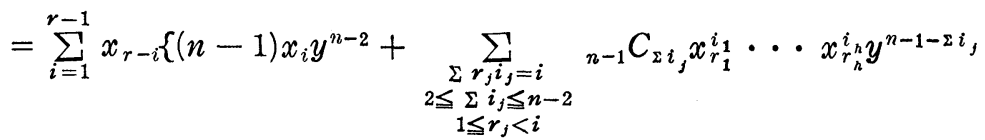

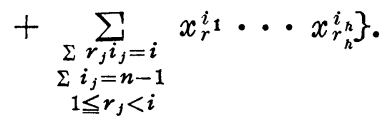

Hence,

$$
\begin{aligned}
& x_{r}^{\prime}(n)=x_{r} y^{n-1}+x_{r}^{\prime}(n-1) y+\sum_{i=1}^{r-1} x_{r-i} x_{i}^{\prime}(n-1) \\
& =n x_{r} y^{n-1}+\sum_{i=1}^{r-1}{ }_{n} C_{2} x_{r-i} x_{i} y^{n-2} \\
& +\sum_{\substack{\sum r_{j} i_{j}=r \\
3 \leqq i_{j} \leqq n-1}} C_{\Sigma i_{j}} x_{r_{1}}^{i_{1}} \cdots x_{r_{h}^{n}}^{i_{h}} y^{n-\Sigma i_{j}} \\
& +\sum_{\substack{\sum r_{i} i_{j}=r \\
i_{j}=n \\
1 \leqq r_{j}<r}} x_{r_{1}}^{i_{1}} \cdots x_{r_{n}^{n}}^{i_{n}} \\
& =n x_{r} y^{n-1} \\
& +\sum_{\substack{\sum \leqq r_{j} i_{j}=r \\
2 \leqq i_{j} \leqq n \\
1 \leqq r_{j}<r}} C_{\Sigma i_{j}} x_{r_{1}}^{i_{1}} \cdots x_{r_{n}^{n}}^{i_{n}} y^{n-\Sigma i_{j}} .
\end{aligned}
$$

This ends the proof.

Corollary 1. Let $T$ be the commutative polynomial ring $k\left[y, x_{1}, \cdots, x_{s}\right]$. Let $x_{r}^{\prime}$ be the mapping of positive integers into $T$ satisfying the conditions 1), 2) and 3) in Lemma 1. Then

$$
\begin{aligned}
x_{r}^{\prime}(n)= & n x_{r} y^{n-1} \\
& +\sum-\frac{\left(\sum i_{j}\right) !}{\Pi\left(i_{j} !\right)}{ }_{n} C_{\Sigma i_{j}} x_{r_{1}}^{i_{1}} \cdots x_{r_{h}^{n}}^{i_{n}} y^{n-\Sigma i_{j}},
\end{aligned}
$$


where the sum is taken over all sets $\left\{r_{1}, \cdots, r_{i} ; i_{1}, \cdots, i_{h}\right\}$ such that $\sum_{j=1}^{h} r_{j} i_{j}=r$, $2 \leqq \sum_{j=1}^{h} i_{j} \leqq n$ and $1 \leqq r_{1}<\cdots<r_{h}<r . \quad$ Moreover if $r$ is not divisible by $p$, then $x_{r}^{\prime}(p)=0$ and if $r=m p$, where $m$ is a positive integer, then $x_{m p}^{\prime}(p)=x_{m}^{p}$.

Proof. The first part is trivial by Lemma 1 . If $1 \leqq r<p$, then $\sum r_{j} i_{j}=r<p$. Therefore ${ }_{p} C_{\Sigma i_{j}} \equiv 0(\bmod p)$.

We assume $m p<r<(m+1) p$. If $\sum i_{j}<p$, then ${ }_{p} C_{\Sigma i_{j}} \equiv 0(\bmod p)$. If $\Sigma i_{j}=p$, by $\sum r_{j} i_{j}=r$, we have $i_{j}<p$. Hence $\frac{p !}{\Pi\left(i_{j} !\right)} \equiv 0(\bmod p)$.

Next we assume $r=m p$. If $\sum i_{j}<p$, then ${ }_{p} C_{\Sigma i_{j}} \equiv 0(\bmod p)$. If $\sum i_{j}=p$ and $i_{j}<p$, then $\frac{p !}{\Pi\left(i_{j} !\right)}=0(\bmod p)$. If $i_{1}=p$, then $r_{1}=m$. This ends the proof.

Remark 1. In Lemma 1, if the condition (2) is defined for $n<p$, then the condition (3) is defined for $n \leqq p$. Therefore Lemma 1 and Corollary 1 are true for $n \leqq p$ and $r>1$.

3. Let $K$ be an inseparable extension field over $k$ such that there exists an inseparable algebraic element $\theta$ of exponent $\alpha$ such that $\theta$ is not contained in $k\left(K^{p}\right)$. Let $f(X)=X^{\beta p \alpha}-a_{\beta-1} X^{\beta-1 p \alpha}-\cdots-a_{1} X^{p \alpha}-a_{0}$ be the minimum polynomial of $\theta$ over $k$. Then there exists $a_{i} \neq 0,1 \leqq i \leqq \beta$, such that $i$ is not divisible by $p$ (where $a_{\beta}=1$ ).

Let $\varphi$ be a derivation of $K$ over $k$ such that $\varphi(\theta)=1$ (see [3]). Let $f_{t}$ be the one-parameter family of deformations of $K$ constructed from $\varphi$ in [1], i.e.,

$$
f_{t}(a, b)=a b+t F_{1}(a, b)+t^{2} F_{2}(a, b)+\cdots,
$$

where $F_{1}=S q_{p} \varphi$.

Lemma 2. Let $f_{t}$ be as above. Then

$$
F_{i}\left(\theta, \theta^{n}\right)=0,
$$

for $i>1$. And if $a \in \operatorname{ker} \varphi$, then

$$
F_{i}(a, b)=0,
$$

for every $b \in K$ and $i \geqq 1$. 
Proof. Let $e_{0}(t \varphi)$ be as in [1,p 72], i.e., $e_{0}(t \varphi)$ is the power series of $t \varphi$ with coefficients in $k$ such that the constant term is 1 and

$$
\begin{aligned}
& e_{0}(t \varphi)\left[e_{0}^{-1}(t \varphi)(a) \cdot e_{0}^{-1}(t \varphi)(b)\right] \\
& \quad=a b+t^{p} F_{1}(a, b)+t^{2 p} F_{2}(a, b)+\cdots,
\end{aligned}
$$

for all $a, b \in V_{s}$. Therefore $F_{i}$ is expressed in the form

$$
\sum_{j=0}^{i p} a_{i j} \varphi^{i p-j} \cup \varphi^{j}, a_{i j} \in k
$$

Hence, for $i>1$, we have

$$
\begin{aligned}
F_{i}\left(\theta, \theta^{n}\right) & =a_{i{ }_{i p-1}} \varphi^{i p-1}\left(\theta^{n}\right)+a_{i i p} \theta \varphi^{i p}\left(\theta^{n}\right) \\
& =0 .
\end{aligned}
$$

On the other hand, if $a \in \operatorname{ker} \varphi$, then $e_{0}^{-1}(t \varphi)(a)=a, e_{0}(t \varphi)(a b)=a e_{0}(t \varphi)(b)$ and therefore $e_{0}(t \varphi)\left[e_{0}^{-1}(t \varphi)(a)\right.$. $\left.\quad e_{0}^{-1}(t \varphi)(b)\right]=a b$. Hence, for $i \geqq 1 \quad F_{i}(a, b)=0$. This ends the proof.

Let

$$
\Phi_{t}=1+t \varphi_{1}+t^{2} \varphi_{2}+\cdots
$$

be a non-singular linear mapping of $V_{s}$ onto itself. If we set

$$
\Phi_{t}^{-1}=1+t \lambda_{1}+t^{2} \lambda_{2}+\cdots \cdot
$$

then we have $\lambda_{r}=-\sum_{i=0}^{r-1} \lambda_{i} \phi_{r-i}=-\sum_{i=0}^{r-1} \varphi_{r-i} \lambda_{i}$, where $\lambda_{0}=1$.

Lemma 3. If we set

$$
\begin{aligned}
& \Phi_{t}^{-1}\left(\Phi_{t}(a) \cdot \Phi_{t}(b)\right) \\
& \quad=a b+t G_{1}(a, b)+t^{2} G_{2}(a, b)+\cdots,
\end{aligned}
$$

then $G_{i}$ satisfies the following conditions;

1) $G_{1}=\delta \varphi_{1}$.

2) For $r \geqq 2$.

$$
G_{r}=\delta \varphi_{r}+\sum_{i=1}^{r-1}\left(\varphi_{r-i} \cup \varphi_{i}-\varphi_{r-i} G_{i}\right)
$$

Proof. 1) is trivial. We may assume $r \geqq 2$.

Then 


$$
\begin{aligned}
G_{r}(a, b)= & \sum_{j=0}^{r} \lambda_{j}\left(\sum_{i=0}^{r-j} \varphi_{i}(a) \varphi_{r-j-i}(b)\right) \\
= & \lambda_{0}\left(\sum_{i=0}^{r} \varphi_{i}(a) \varphi_{r-i}(b)\right) \\
& -\left(\varphi_{1} \lambda_{0}\right)\left(\sum_{i=0}^{r-1} \varphi_{i}(a) \varphi_{r-1-i}(b)\right)-\cdots \\
& -\left(\varphi_{j} \lambda_{0}+\cdots+\varphi_{1} \lambda_{j-1}\right)\left(\sum_{i=0}^{r-j} \varphi_{i}(a) \varphi_{r-j-i}(b)\right) \\
& -\cdots-\left(\varphi_{r} \lambda_{0}+\cdots+\varphi_{1} \lambda_{r-1}\right)(a b) \\
= & \delta \varphi_{r}(a, b)+\sum_{i=1}^{r-1} \varphi_{i}(a) \varphi_{r-i}(b) \\
& -\varphi_{1}\left[\lambda_{0}\left(\sum_{i=0}^{r-1} \varphi_{i}(a) \varphi_{r-1-i}(b)\right)+\cdots+\lambda_{r-1}(a b)\right]-\cdots \\
& -\varphi_{j}\left[\lambda_{0}\left(\sum_{i=0}^{r-j} \varphi_{i}(a) \varphi_{r-j-i}(b)\right)+\cdots+\lambda_{r-j}(a b)\right]-\cdots \\
& -\varphi_{r-1}\left[\lambda_{0}\left(a \varphi_{1}(b)+\varphi_{1}(a) b\right)+\lambda_{1}(a b)\right] \\
= & \left\{\delta \varphi_{r}+\sum_{i=1}^{r-1}\left(\varphi_{i} \cup \varphi_{r-i}-\varphi_{i} G_{r-i}\right)\right\}(a, b) .
\end{aligned}
$$

This ends the proof.

Nowx we assume $f_{t}$ is trivial, i.e., there exists $\Phi_{t}=1+t \varphi_{1}+t^{2} \varphi_{2}+\cdots$ such that

$$
f_{t}(a, b)=\Phi_{t}^{-1}\left(\Phi_{t} a \cdot \Phi_{t} b\right)
$$

Then $G_{i}=F_{i}$ for all $i$. In [4] we proved $\varphi_{1}\left(\theta^{n}\right)=n \theta^{n-1} \varphi_{1}(\theta)+m \theta^{n-p}$ for $m p \leqq n<(m+1) p$.

Proposition 1. If $f_{t}$ is trivial, then $\varphi_{r}$ satisfies the following consitions;

1) $\varphi_{r}(1)=0$, for $r \geqq 1$.

2) $\varphi_{p} m\left(\theta^{n p^{m+1}}\right)=n \theta^{(n-1) p^{m+1}}$

3) If $r(>1)$ is not divisible by $p$, then $\varphi_{r}\left(\theta^{p}\right)=0$.

4) If $r$ is not divisible by $p^{m}(m>0)$, then $\varphi_{r}\left(\theta^{p^{m+1}}\right)=0$.

Proof. 1). We shall prove by induction on $r$. If $r=1$, then this is trivial. By Lemma 2, $G_{r}(1,1)=0$ for $r \geqq 1$. Thereofre, by Lemma 3, $\delta \varphi_{r}(1,1)=0$. Hence $\varphi_{r}(1)=0$.

3). $\varphi_{1}\left(\theta^{n}\right)=n \theta^{n-1} \varphi_{1}(\theta)$ for $n<p$, and by Lemma $2, G_{i}\left(\theta, \theta^{n}\right)=0$ for $i>1$. On the other hand $G_{1}\left(\theta, \theta^{n}\right)=0$ 
or -1 for $n \leqq p$. Therefore $\varphi_{r-1} G_{1}\left(\theta, \theta^{n}\right)=0$. Hence we have, by Lemma 3,

$$
\begin{aligned}
\varphi_{r}\left(\theta^{n}\right)= & \theta^{n-1} \varphi_{r}(\theta)+\theta \varphi_{r}\left(\theta^{n-1}\right) \\
& +\sum_{i=1}^{r-1} \varphi_{i}(\theta) \varphi_{r-i}\left(\theta^{n-1}\right)
\end{aligned}
$$

Hence if we set $x_{i}=\varphi_{i}(\theta), \quad x_{i}^{\prime}(n)=\varphi_{i}\left(\theta^{n}\right)$ and $y=\theta$, then, by Remark 1, $\varphi_{r}\left(\theta^{p}\right)=0$, where $r$ is not divisible by $p$ and $r>1$.

2) and 4). By [4, Lemma 2], $\varphi_{1}\left(\theta^{n p}\right)=n \theta^{(n-1) p}$.

We shall prove by induction on $m$.

i) The case $m=1$. By Lemma 2, $G_{i}\left(\theta^{p}, \theta^{n p}\right)=0$.

By Lemma 3, we have

$$
\begin{aligned}
\varphi_{r}\left(\theta^{n p}\right)= & \theta^{(n-1) p} \varphi_{r}\left(\theta^{p}\right)+\theta^{p} \varphi_{r}\left(\theta^{(n-1) p}\right) \\
& +\sum_{i=1}^{r-1} \varphi_{i}\left(\theta^{p}\right) \varphi_{r-i}\left(\theta^{(n-1) p}\right) .
\end{aligned}
$$

Set $x_{i}=\varphi_{i}\left(\theta^{p}\right), \quad x_{i}^{\prime}(n)=\varphi_{i}\left(\theta^{n p}\right)$ and $y=\theta^{p}$. Then, by Corollary 1 , if $r$ is not divisible by $p$, then $x_{r}(p)=\varphi_{r}\left(\theta^{p^{2}}\right)=0$. If $1<i<p$, then $x_{i}=\varphi_{i}\left(\theta^{p}\right)$ $=0$ by 3 ). Therefore, by Corollary 1 , we have

$$
\begin{aligned}
\varphi_{p}\left(\theta^{n p^{2}}\right) & =x_{p}(n p) \\
& ={ }_{n p} C_{p} x_{1}^{p} y^{(n-1) p}=n\left\{\varphi_{1}\left(\theta^{p}\right)\right\}^{p} \theta^{(n-1) p^{2}} \\
& =n \theta^{(n-1) p^{2}} .
\end{aligned}
$$

ii) The case $m>1$. By $G_{i}\left(\theta^{p^{m}}, \theta^{n p^{m}}\right)=0$.

Hence we have

$$
\begin{aligned}
\varphi_{r}\left(\theta^{n p^{m}}\right)= & \theta^{p^{m}} \varphi_{r}\left(\theta^{(n-1) p^{m}}\right)+\theta^{(n-1) p^{m}} \varphi_{r}\left(\theta^{p^{m}}\right) \\
& +\sum_{i=1}^{r-1} \varphi_{i}\left(\theta^{p^{m}}\right) \varphi_{r-i}\left(\theta^{(n-1) p^{m}}\right)
\end{aligned}
$$

Set $x_{i}=\varphi_{i}\left(\theta^{p^{m}}\right), x_{i}^{\prime}(n)=\varphi_{i}\left(\theta^{n p^{m}}\right)$ and $y=\theta^{p^{m}}$. If $r$ is not divisible by $p$, then $x_{r}^{\prime}(p)=\varphi_{r}\left(\theta^{p}\right)=0$ and if $r=u p^{v}$, where $u$ is not divisible by $p$ and $0<v<m$, then $\varphi_{r}\left(\theta^{p^{m+1}}\right)=x_{r}^{\prime}(p)=\left\{x_{u p^{v-1}}\right\}^{p}=\left\{\varphi_{u p^{v-1}}\left(\theta^{p^{m}}\right)\right\}^{p}=0$. Hence 4) was proved. On the other hand, if $i$ is not divisible by $p^{m-1}$, then $x_{i}=\varphi_{i}\left(\theta^{p^{m}}\right)=0$ by the assumption of induction. Therefore we have 


$$
x_{p^{m}}^{\prime}(n p)=\sum \frac{\left(\sum i_{j}\right)}{\Pi\left(i_{j}\right)}{ }_{n p} C_{\Sigma i_{j}} x_{r_{1}}^{i_{1}} \cdots x_{r_{h}}^{i_{h}} y^{n p-\Sigma n_{j}}
$$

where the sum is taken over all sets $\left\{r_{1}, \cdots, r_{h} ; i_{1}, \cdots, i_{h}\right\}$ such that $\sum_{j=1}^{h} r_{j} i_{j}=p^{m}, 2 \leqq \sum i_{j} \leqq n p, 1<r_{1}<\cdots<r_{h}<p^{m}$ and every $r_{j}$ is divisible by $p^{m-1}$. We may set $r_{j}=u_{j} p^{m-1}$, where $0<u_{j}<p$. Hence we have $\sum_{j=1}^{h} u_{j} i_{j}=p$ and we may assume $\sum_{j=1}^{h} i_{j} \leqq p$. If $\sum_{j=1}^{h} i_{j}<p$, then ${ }_{n p} C_{\Sigma i_{j}} \equiv 0$ $(\bmod p)$, and if $\sum_{j=1}^{h} i_{j}=p$ and $i_{j}<p$ for all $j$, then $\frac{\left(\sum i_{j}\right) !}{\Pi\left(i_{j} !\right)} \equiv 0(\bmod p)$. Therefore we have

$$
\begin{aligned}
\varphi_{p^{m}}\left(\theta^{n p^{m+1}}\right) & =x_{p^{m}}^{\prime}(n p)={ }_{n p} C_{p} x_{p^{m-1}}^{p} y^{(n-1) p} \\
& =n\left\{\varphi_{p^{m-1}}\left(\theta^{p^{m}}\right)\right\}^{p} \theta^{(n-1) p^{m+1}} \\
& =n \theta^{(n-1) p^{m+1}} .
\end{aligned}
$$

This completes the proof.

By Proposition 1, we have

$$
\varphi_{p^{\alpha-1}}\left(\theta^{\beta p^{\alpha}}\right)=\beta \theta^{(\beta-1) p^{\alpha}} .
$$

On the other hand,

$$
\varphi_{p^{\alpha-1}}\left(\theta^{\beta p^{\alpha}}\right)=\varphi_{p^{\alpha-1}}\left(\sum_{i=0}^{\beta-1} a_{i} \theta^{i p^{\alpha}}\right)=\sum_{i=1}^{\beta-1} i a_{i} \theta^{(i-1) p^{\alpha}} .
$$

Therefore $\beta \equiv 0(\bmod p)$ and if $a_{i} \neq 0$, then $i \equiv 0(\bmod p)$. Hence $\theta$ is an inseparable element of exponent $>\alpha$ over $k$. This is contradiction, and we have obtained the following.

THEOREM. Let $K$ be an extension field of a field $k$ of characteristic $p \neq 0$. If there exists an inseparable algebraic element such that it is not contained in $k\left(K^{p}\right)$, then $K$ is not rigid, and a non-trivial integrable element of $H_{c}^{2}(K, K)$ is found in the image of $S q_{p}$.

Remark 2. Let $K$ be an algebraic extension field of a field $k$. By [1, p 79, Cor. 2] and the above theroem, $K$ is separable over $k$ if and only if considered as an algebra over $k, K$ is rigid.

\section{REFERENCES}

[ 1] M. Gerstenhaber: On the deformation of rings and algebras, Ann. of Math. 79 (1964), 59-103. 
[2] D.K. Harrison: Commutative algebras and cohomology, Trans. Amer. Math. Soc., 104 (1962), 191-204.

[ 3 ] N. Jacobsen: Lectures in abstract algebra III, Van Nastrand, 1964.

[4] H. Kimura: Existence of non-trivial deformations of some inseparable extension fields, Nagoya Math. J. 31 (1968), 37-40.

Nagoya University 